\title{
Necessidade de rede de apoio para usuários com nefrostomia: Relato de experiência
}

\author{
Need for a support network for users with nephrostomy: Experience report \\ Necesidad de una red de apoyo para usuarios con nefrostomía: Informe de experiencia
}

Recebido: 24/05/2021 | Revisado: 02/06/2021 | Aceito: 05/06/2021 | Publicado: 17/06/2021

\author{
Ana Maria Pagliarini \\ ORCID: https://orcid.org/0000-0003-4585-4846 \\ Universidade Federal do Rio Grande do Sul, Brasil \\ E-mail: amp.pagliarini5@gmail.com \\ Rosaura Soares Paczek \\ ORCID: https://orcid.org/0000-0002-4397-1814 \\ Hospital de Clínicas de Porto Alegre, Brasil \\ E-mail: rspaczek@gmail.com \\ Rafaela Garbini Casarin \\ ORCID: https://orcid.org/0000-0002-3263-6201 \\ Universidade Federal do Rio Grande do Sul, Brasil \\ E-mail: rafaelagcasarin@gmail.com \\ Ana Karina Silva da Rocha Tanaka \\ ORCID: https://orcid.org/0000-0003-2488-3656 \\ Universidade Federal do Rio Grande do Sul, Brasil \\ E-mail: anakarinatanaka@gmail.com \\ Lisiane Paula Sordi Matzenbacher \\ ORCID: https://orcid.org/0000-0002-1407-7717 \\ Hospital de Clínicas de Porto Alegre, Brasil \\ E-mail: 1sordi@hcpa.edu.br \\ Débora Machado Nascimento do Espírito Santo \\ ORCID: https://orcid.org/0000-0003-0533-0335 \\ Hospital de Clínicas de Porto Alegre, Brasil \\ E-mail: dsanto@hcpa.edu.br \\ Carina Galvan \\ ORCID: https://orcid.org/0000-0003-2111-5432 \\ Hospital de Clínicas de Porto Alegre, Brasil \\ E-mail: cgalvan@hcpa.edu.br \\ Nathalia Lima Pereira \\ ORCID: https://orcid.org/0000-0002-5181-7550 \\ Escola de Saúde Pública, Brasil \\ E-mail: nathilimap@gmail.com
}

\begin{abstract}
Resumo
Objetivo: relatar a experiência de acadêmicas de enfermagem e enfermeiras no atendimento a usuários com nefrostomia. Metodologia: estudo tipo relato de experiência realizado em 2021 num centro de referência para atendimento a usuários com estomias numa cidade do sul do Brasil, sendo selecionados dados sobre usuários com nefrostomia, analisando qual o motivo da procura por atendimento com a enfermeira estomaterapeuta, durante a consulta de enfermagem. Resultados: foram observadas dificuldades na realização do autocuidado associados a troca do sistema coletor, pois a localização da estomia não favorece a fixação adequada da bolsa frente a tentativa do próprio usuário realizar a troca do equipamento coletor, dependendo sempre de uma outra pessoa para realizar essa troca. Os usuários do serviço relatam que mesmo com auxílio de espelhos, a manobra a ser utilizada impossibilita o posicionamento adequado do equipamento coletor, favorecendo vazamentos e diminuindo o tempo útil das bolsas coletoras. Considerações finais: o profissional enfermeiro deve avaliar a pessoa com nefrostomia, as condições do estoma e da pele periestomal, prescrevendo o equipamento coletor e adjuvantes mais adequados para cada caso, a posição da bolsa coletora deve proporcionar o esvaziamento da mesma pelo próprio usuário, sendo necessário realizar treinamento de familiar para auxiliar nas trocas.
\end{abstract}

Palavras-chave: Nefrostomia; Enfermagem; Autocuidado.

\begin{abstract}
Objective: to report the experience of nursing students and nurses in the care of users with nephrostomy Method: an experience report study carried out in 2021 in a reference center for stoma patients in a city in southern Brazil, and selected data on users with nephrostomy, analyzing the reason for seeking care with the stoma therapist nurse during the nursing consultation. Results: difficulties were observed in performing self-care associated with the change of the collecting system, because the location of the ostomy does not favor the adequate fixation of the bag in front of the
\end{abstract}


user's own attempt to change the collecting equipment, always depending on another person to perform this change. The service users report that even with the aid of mirrors, the maneuver to be used makes it impossible to properly position the collection equipment, favoring leaks and decreasing the useful time of the collection bags. Final considerations: The professional nurse must evaluate the person with nephrostomy, the conditions of the stoma and the peristomal skin, prescribing the collecting equipment and adjuvants best suited for each case, the position of the collecting bag must allow its emptying by the user, and it is necessary to train family members to assist in the exchanges.

Keywords: Nephrostomy; Nursing; Self care.

\section{Resumen}

Objetivo: reportar la experiencia de estudiantes de enfermería y enfermeras en el cuidado de usuarios con nefrostomía Método: estudio experiencia-informe realizado en 2021 en un centro de referencia para el cuidado de usuarios con ostomías en una ciudad del sur de Brasil, con datos de usuarios con nefrostomía, analizando el motivo de consulta con la enfermera estomaterapeuta durante la consulta de enfermería. Resultados: se observaron dificultades en la realización del autocuidado asociadas al cambio del sistema de recolección, ya que la ubicación de la ostomía no favorece la correcta fijación de la bolsa ante el intento del propio usuario de cambiar el equipo de recolección, siempre dependiendo de otra persona para realizar este reemplazo. Los usuarios del servicio informan que incluso con la ayuda de espejos, la maniobra a realizar imposibilita la correcta colocación del equipo recolector, favoreciendo las fugas y reduciendo el tiempo útil de las bolsas recolectoras. Consideraciones finales: El profesional enfermero debe evaluar a la persona con nefrostomía, las condiciones del estoma y de la piel periestomal, prescribiendo el equipo de recolección y adyuvantes más adecuados para cada caso, la posición de la bolsa de recolección debe permitir su vaciado por parte del usuario, siendo necesario realizar un entrenamiento familiar para ayudar en los cambios.

Palabras clave: Nefrostomía; Enfermería; Autocuidado.

\section{Introdução}

A nefrostomia percutânea é um procedimento que consiste na realização de uma comunicação direta do rim com o meio exterior através de uma sonda flexível introduzida, com auxílio de equipamento de imagem, por um orifício na pele. Thomas Hiller descreveu o acesso renal percutâneo pela primeira vez em 1865, a próxima descrição aparece na literatura apenas em 1955, onde Goddwin descreveu um acesso renal percutâneo em rim hidronefrótico (Zapato-González, CamachoCastro, García, Reyna-Bulnes, \& Robles-Scott, 2014; Kamei et al., 2020). Esta técnica visa o acesso ao sistema urinário objetivando a drenagem de urina do rim, geralmente impossibilitado por obstrução supravesical, que pode ser de natureza mecânica ou alterações anatômicas, diminuindo a ocorrência de hidronefrose e de comprometimento renal (Fernández-Cacho \& Ayesa-Arriola, 2019; Meira et al, 2019). Por estabelecer um acesso direto ao sistema urinário, outras indicações da nefrostomia são: dissolução de cálculos, infusão de quimioterápicos, antibiótico ou antifúngicos, desvio do fluxo para otimizar o tratamento de fístulas, além de drenagem de coleções perirrenais (Meira et al, 2019).

Dadas as características de facilidade do procedimento, resolutividade e baixa incidência de complicações, esta técnica tem sido amplamente utilizada, podendo ter caráter temporário ou permanente. Sua instalação ocorre por punção direta, na região lombar, com anestesia local e auxílio de ultrassonografia (Fernández-Cacho \& Ayesa-Arriola,2019), tomografia computadorizada ou fluoroscopia (Meira et al, 2019), o cateter é adaptado a uma bolsa coletora. A instalação da nefrostomia é considerada um procedimento minimamente invasivo devido a possibilidade de ser realizada sob efeito de sedação, sendo dispensado a utilização de recursos anestésicos maiores. O êxito do procedimento está associado a diversas condições, que variam desde o estado geral do usuário, à situação e anatomia do rim (Fernández-Cacho \& Ayesa-Arriola,2019; Tibana et al,2019).

Quando necessária desobstrução urgente no sistema urinário, em razão de malignidade, a nefrostomia é o método mais indicado. Porém, o uso da bolsa coletora é um fator que causa desconforto e reduz a qualidade de vida destes usuários (Santos, Tibana, Marchiori, \& Nunes, 2020). A pessoa com estomia tem direito a receber atenção especializada, deve receber as bolsas coletoras, assim como a prescrição de cuidados por enfermeiros (Sousa, Rosa, Campos, \& Ginaldi, 2021). Salienta-se que o predomínio de complicações em pessoas com estomia está relacionado aos cuidados e uso de dispositivos inadequados, 
necessitando de profissionais especializados no manejo de estomias principalmente na rede de atenção básica de saúde (Stegensek-Mejía, Murad-Robles, González-Mier, López-Hernández, \& Sánchez-Ojeda, 2017).

A consulta de enfermagem, atividade privativa do enfermeiro, regulamentada pelo COFEN pela lei 7498/86, tem como objetivo identificar as principais demandas, prescrevendo e implementando medidas de enfermagem para promoção, proteção e recuperação da saúde (Brasil,1986). O sucesso da consulta está ligado ao conhecimento clínico relacionado ao enfermeiro-usuário, estabelecendo uma comunicação efetiva, tornando a consulta mais resolutiva (Mota, Gomes, \& Petuco, 2016). Através destas consultas, há o acompanhamento e apoio de forma singular e individualizada de acordo com a demanda do usuário, promovendo durante o processo de transição e adaptação ao estoma, a capacitação para o autocuidado e melhora da qualidade de vida (Miranda, Carvalho, \& Paz, 2018).

Apesar da realização de nefrostomia ser cada vez mais frequente, encontramos poucos artigos que abordam o autocuidado em pessoas com nefrostomia. Para tanto, este estudo tem como objetivo relatar experiências no atendimento de enfermagem a usuários de nefrostomia e o que os leva a buscar o serviço especializado para realizar a troca do dispositivo.

\section{Metodologia}

Trata-se de um relato das experiências de acadêmicas de enfermagem e enfermeiras, vivenciadas em um centro de referências para atendimento a usuários com estomias numa cidade do sul do Brasil, realizado em abril de 2021. As consultas são realizadas através de agendamento prévio, ou demanda espontânea de segunda a sexta-feira. A amostra foi obtida através da análise dos prontuários dos usuários com nefrostomia cadastrados para aquisição de equipamentos para estomias e incontinências, verificando o motivo da procura por atendimento com a enfermeira estomaterapeuta. Dados sociodemográficos foram obtidos através da mesma temática. Foram incluídos os usuários com nefrostomia que consultaram neste período, sendo excluídos os usuários que estavam com dados incompletos no prontuário ou que compareceram ao serviço somente para obtenção de material. A amostra foi constituída por doze usuários que consultaram dentro do período analisado. A pesquisa foi aprovada pelo Comitê de Ética em Pesquisa da Secretaria Municipal de Saúde da Prefeitura de Porto Alegre- RS CAAE $n^{\circ}$ 17789319.6.0000.5338 sob parecer $\mathrm{n}^{\mathrm{o}} 3.530 .685$.

\section{Resultados}

O estudo foi realizado num centro de referência de estomaterapia de uma cidade do sul do Brasil, que funciona de segunda a sexta-feira, atendendo usuários com estomas intestinais, urinários e lesões de pele, residentes na área de abrangência, que é determinada pelo município. Para recebimento dos materiais deve ser realizado cadastro no centro de referência, sendo necessário apresentar documentos do usuário e do responsável, comprovante de residência e laudo médico. São agendadas consultas com a enfermeira estomaterapeuta para avaliação e adequação do material a ser utilizado.

O serviço de estomaterapia possui 617 pessoas com estomias cadastradas, dentre estas doze com nefrostomia, o que corresponde 1,94\%, sendo 58,33\% (7) do sexo feminino, e 41,66\% (5) do sexo masculino. Quanto à idade, 33,33\% (4) com idade entre 40 e 60 anos e $66,66 \%$ (8) maiores de 60 anos.

Durante o período analisado foram realizadas 29 consultas de enfermagem com usuários com nefrostomia, tendo o número de consultas variando de uma a oito, onde o principal motivo para atendimento foi para realizar a troca de bolsa. Cabe ressaltar que também foram realizadas consultas de enfermagem para avaliação de dermatites, baixa durabilidade do equipamento coletor e tratamento de lesões de pele advindas do uso de dispositivo de nefrostomia. As complicações mais frequentes verificadas nesses atendimentos foram granulomas e dermatite periestomal. Dos doze usuários com nefrostomia cadastrados atualmente no centro de referência do estudo, dois estão realizando as trocas do equipamento coletor somente no 
serviço, com dias agendados com a enfermeira estomaterapeuta. Estes,também estão em acompanhamento oncológico, portanto as necessidades em saúde perpassam o cuidado com a nefrostomia. As trocas de saberes referentes ao dispositivo implementado, complementar à função renal de um indivíduo, muitas vezes previamente saudável, trazendo confiança a partir da necessidade de cuidado especializado, faz com que sejam criados vínculos com os profissionais de saúde que prestam essa assistência.

Nos atendimentos de enfermagem realizados a usuários com nefrostomia foram observadas as dificuldades da realização do autocuidado, frequentemente atreladas a localização anatômica da estomia. Por tratar-se de um cateter inserido na região lombar há dificuldade de acesso para a troca do sistema coletor. Tal situação não favorece a fixação adequada da bolsa frente a tentativa do próprio usuário realizar a troca do dispositivo, dependendo sempre de uma outra pessoa para realizar essa troca. Há também o relato que mesmo com auxílio de espelhos, a manobra a ser utilizada impossibilita o posicionamento adequado do equipamento coletor, o que ocasiona vazamentos e diminui o tempo útil dos mesmos.

A ausência de rede de apoio familiar e, por vezes, da unidade básica de saúde, também contribui para que o usuário se desloque até o centro de referência. É observada muitas vezes o não comparecimento de familiar às consultas de enfermagem, não sendo possível a orientação quanto aos cuidados por parte da enfermeira estomaterapeuta. Neste caso, ocorre a procura pelo serviço com maior frequência, dada a impossibilidade de realizar este procedimento de forma autônoma. Quando o equipamento coletor é trocado no centro de saúde, observa-se que o mesmo tem durabilidade de 8 dias e raramente ocorrem complicações na pele periestomal.

\section{Discussão}

A realização de um procedimento que resulte na utilização de um estoma tem como finalidade a manutenção das funções de eliminações necessárias para o adequado funcionamento do organismo. $\mathrm{O}$ uso de um dispositivo com a finalidade de excreção impacta na percepção da autoimagem e na qualidade de vida do indivíduo. Mesmo que a utilização do estoma represente a manutenção da vida, a necessidade do uso de tal dispositivo leva o usuário, por vezes, a questionar o custo de manter-se vivo. Dentre os temores vivenciados pelos usuários estão: estar fora dos padrões da normalidade, o risco da percepção por partes de outros do uso do dispositivo, risco de vazamento ou descolamento e possibilidade de limitações físicas são os mais citados (Brasil, 1986). Tais temores são relatados com frequência durante os atendimentos, principalmente no período logo após a instalação do estoma. O maior temor, mencionado, é de que o dispositivo descole e que venha a ocorrer vazamento de conteúdo na presença de outrem.

A condição de estar com um estoma leva o indivíduo a fazer um reajuste de sua rotina e hábitos de vida. Mesmo aqueles usuários que possuem um nível maior de aceitação almejam a reversão da condição o mais breve possível. Viver com um estoma requer o desenvolvimento de habilidades para manutenção adequada deste e do dispositivo coletor (Coelho, Santos, \& Poggetto, 2013). No processo de adaptação à nova condição, o usuário com estoma acaba por buscar uma rede de apoio que o auxiliará na adaptação e na superação dos desafios que possam surgir. $\mathrm{O}$ apoio familiar, a orientação profissional e a vivência de outras pessoas que estão nas mesmas condições agregam na recuperação, trazendo um grau maior de confiança. Nesse contexto, o enfermeiro e a rede de apoio eficaz possibilitam maior segurança, sendo fundamentais para a reabilitação e adaptação à nova condição (Albuquerque, Pinheiro, Linhares \& Guedes, 2016). Ações educativas de enfermagem para o conhecimento de sua situação influenciam na adaptação, impactando positivamente e tornando o usuário parte responsável do seu cuidado (Coelho et al, 2013).

O cuidado pautado no conhecimento científico, inicia na fase pré-operatória e se estende até o retorno do usuário à unidade básica de saúde. Torna-se necessário garantir que a aquisição e a padronização de equipamentos coletores e adjuvantes de proteção e segurança atendam às necessidades individuais desses usuários (Almeida \& Silva, 2019; Bandeira, Kolankiewicz, 
Alievi, Trindade \& Loro, 2020; Moreira, Souza, Oliveira, Melo \& Cerqueira, 2017).O Ministério da Saúde, por meio da Portaria da Secretaria de Atenção à Saúde (SAS/MS n 400, de 16 de novembro de 2009), regulamenta as Políticas de Saúde das Pessoas Ostomizadas. Os serviços de atenção à saúde devem estabelecer fluxos e mecanismos de referência e contrarreferência, prezando pela adequada e completa assistência das pessoas com estomias (Brasil, 2009).

No cenário de adaptação e convivência com o estoma, o profissional da saúde tem importância significativa na obtenção da capacidade de autocuidado. Alterações físicas, psicológicas, fisiológicas e sociais estão atreladas a este processo. O preparo do paciente previamente à realização da estomia é fundamental para adaptação a sua nova condição de vida, contribuindo para uma melhor qualidade de vida (Miranda et al., 2018). Há necessidade de um olhar individualizado frente à nova condição para que, através de um acompanhamento ambulatorial ou até domiciliar, seja realizada uma análise das necessidades e vivências de cada um e do seu processo de reabilitação, sendo necessária uma reflexão do profissional frente às fragilidades nessa nova situação (Paczek, Engelmann, Perini, Aguiar, \& Duarte, 2020). As complicações periestomais mais comuns são os danos à pele. Uma pele periestomal saudável é imprescindível para uma boa aderência da bolsa coletora, o que evita que o líquido drenado penetre na pele. Os acessórios precisam ser adequados a fim de evitar vazamento de efluentes (O'Flynn,2019). O enfermeiro estomaterapeuta é o profissional capacitado e responsável pela educação deste usuário frente a esta nova realidade (Carter, 2020).

A coerência e confiança passada pelo profissional durante o atendimento, encorajando o usuário ao protagonismo do seu cuidado, proporciona a manutenção da sua qualidade de vida e minimiza o surgimento de complicações. A adesão ao tratamento é parte importante do processo e o estar instrumentalizado faz com que o usuário se encoraje a realizar o seu cuidado de forma ativa (Ribeiro et al, 2019). Observa-se que a orientação sobre a dificuldade para a troca do dispositivo coletor está relacionada ao posicionamento anatômico da mesma, devendo ter alguém capacitado para realizar a troca do dispositivo, onde o usuário deve conhecer o processo de troca, mesmo sem ter condições, torna-o participante ativo de seu autocuidado.

\section{Considerações Finais}

Durante o atendimento aos usuários de nefrostomia, observou-se que aqueles que possuem uma rede de apoio familiar estabelecida, onde há busca de conhecimento no centro de saúde referência por instruções e treinamentos para a manutenção do cuidado domiciliar, as complicações e a frequência de busca por atendimentos têm menor frequência. Em contrapartida, aqueles que não possuem vínculos familiares bem estruturados, com relações conflituosas ou ausência de rede de apoio, há uma busca frequente por atendimento para realizar a troca do dispositivo, e, quando esta ocorre de forma mais espaçada, ao retorno, observa-se maior presença de complicações, como dermatites irritativas ocasionadas por vazamento de conteúdo urinário do dispositivo coletor, causadas por descolamento da placa.

Uma das ações mais frequentemente tomadas na atuação do centro de referência e do enfermeiro estomaterapeuta envolvido no cuidado ao usuário é encorajar e auxiliar aquele usuário sem rede de apoio a vincular-se a alguém próximo, convidando-o a participar desse processo e pedindo auxílio, para que, em uma situação de dificuldade de deslocamento até o centro de saúde. Outra ação é a articulação com a unidade básica de saúde a qual o usuário é adscrito, capacitando a equipe de saúde a prestar esse suporte àquele usuário. No período do presente estudo, essa ação pode ser realizada apenas através de contatos à distância, já que o momento não permitia os treinamentos de forma presencial. Mas cabe ressaltar que ações dessa magnitude já foram realizadas em momentos anteriores, e, que ainda assim, devido ao vínculo de confiança há usuários que não abrem mão de serem atendidos pelo estomaterapeuta. Tal observação, não invalida a percepção de que o usuário de nefrostomia necessita de uma rede de apoio próxima ao seu convívio para que tenha segurança nas suas atividades diárias.

Vale ressaltar que mesmo nos casos em que o usuário possua rede de apoio, cabe ao profissional enfermeiro avaliação 
das condições do estoma e da pele periestomal, prescrevendo o equipamento coletor e adjuvantes mais adequados para cada caso, a posição do equipamento coletor deve proporcionar o esvaziamento do mesmo pelo próprio usuário. Avalia-se também qual a melhor posição da bolsa coletora para facilitar a drenagem e o manuseio da mesma, assim como a frequência das trocas.

As limitações encontradas para realização deste estudo estão ligadas a literatura quanto ao autocuidado de pacientes com nefrostomia, apesar de haver artigos que tratem dessa temática em abundância relacionada ao autocuidado com as estomias intestinais, a literatura relacionada a nefrostomias é menos frequente. Os artigos encontrados que fazem referência a nefrostomia, em sua maioria, são de estudos na área da medicina, associados à instalação do cateter. Outra limitação encontrada, foi o período que vivenciamos uma pandemia, onde a procura pelo serviço pode ter sofrido redução, já que os atendimentos no centro de saúde sofreram alterações por recomendações de isolamento social.

\section{Referências}

Albuquerque, A. F. L. L., Pinheiro, A. K. B., Linhares, F. M. P., \& Guedes, T. G.(2016). Technology for self-care for ostomized women's sexual and reproductive health. Rev Bras Enferm. ,69(6):1099-1106.

Almeida, E. J.,\& Silva, A. L. (2015). Caracterização do Perfil Epidemiológico dos Estomizados em Hospitais da Secretaria de Estado de Saúde do Distrito Federal. Rev Estima, 13(1): 11-16.

Bandeira, L. R., Kolankiewicz, A. C. B., Alievi, M. F.,Trindade, L. F., \& Loro, M. M. (2020). Atenção integral fragmentada a pessoa estomizada na rede de atenção à saúde. Esc. Anna. Nery, 24 (3).

Brasil. (1986). Constituição da República Federativa do Brasil de 1986. (1986). Lei no 7498, de 25 de junho de 1986. Dispõe sobre a Re gulamentação do Exercício da Enfermagem e Dá Outras Providências. http://www.planalto.gov.br/ccivil_03/leis/17498.htm.

Brasil. (2009). Ministério da Saúde. Portaria n 400, de 16 de novembro de 2009. (2009). Estabelece Diretrizes Nacionais para a Atenção à Saúde das Pessoas Ostomizadas. Diário Oficial da União . 2009 ; 01: 41-42. http://bvsms.saude.gov.br/bvs/saudelegis/sas/2009/prt0400_16_11_2009.html

Carter, P. (2020). Recognising the importance of stoma care nurse specialists. Br J Nurs., 26;29(6):S4.

Coelho, A. R., Santos, F. S., \& Poggetto, M. G. (2013). A estomia mudando a vida: enfrentar para viver. Rev Min Enferm. 17(2).

Fernández-Cacho, L. M., \& Ayesa-Arriola, R. (2019). Quality of life, pain and anxiety in patients with nephrostomy tubes. Rev. Latino-Am. Enfermagem, 27:e3191.

Kamei, D. J., Ehlert, R., Dall'Asta, L., Yoshikawa, G. B., Santos, L. S., \& Machado, C. (2020). Comparação dos Resultados Cirúrgicos da Nefrolitotripsia Percutânea entre as Posições Prona e Valdivia-Galdakao. Rev. Méd. Paraná,78(1):32-39 .

Meira, M. S., Barbosa, P. N. V. P., Bitencourt, A. G. V., Almeida, M. F. A., Tyng, C. J., Costa, M,A,F, Góes, A. C. A.,\& Chojniaket, R. (2019). Retrospective analysis of computed tomography-guided percutaneous nephrostomies in cancer patients. Radiol Bras ., 52( 3 ): 148-154.

Miranda, L. S. G., Carvalho, A. A. S., \& Paz, E. P. A. (2018). Qualidade de vida da pessoa estomizada: relação com os cuidados prestados na consulta de enfermagem de estomaterapia. Esc. Anna Nery. , 22( 4 ): e20180075.

Moreira, L. R., Souza, J. C., Oliveira, M. M., Melo, N. S., \& Cerqueira, T. F. (2017). Autocuidado com estomias: compreensão de pacientes hospitalizados acerca das orientações recebidas pela equipe. Rev Enferm, 20(2)

Mota, M. S.,; Gomes, G. C., \& Petuco, V. M.(2016). Repercussions In The Living Process Of People With Stomas. Texto Contexto - Enferm., 25(1), E1260014.

O'Flynn SK. Peristomal skin damage: assessment, prevention and treatment. Br J Nurs. (2019). 14;28(5):S6-S12.

Paczek, R. S., Engelmann, A. I., Perini, G. P., Aguiar, G. P. S. de, \& Duarte, E. R. M. (2020). Perfil de usuários e motivos da consulta de enfermagem em estomaterapia. Rev enferm UFPE on line, 14.

Ribeiro, W. A., Andrade, M., Couto, C. S., Souza, D. M. S., de Morais, M. C., \& Santos, J. A. M (2019). As contribuições do enfermeiro no autocuidado ao paciente estomizado: uma revisão integrativa. Revista Pró-UniverSUS, 10 (1): 72-75.

Santos, R. F. T., Tibana, T. K., Marchiori, E., \& Nunes, T. F. (2020). Inserção anterógrada de cateter duplo J no tratamento de obstruções ureterais malignas: análise retrospectiva dos resultados de uma técnica modificada em um hospital universitário. Radiol Bras, 53 (3).

Sousa, J., Rosa, M., Campos, C., \& Rinaldi, E. (2021). Perfil de usuários estomizados atendidos em um departamento de órteses e próteses. Enfermagem em Foco, 11(4).

Stegensek-Mejía, E. M., Murad-Robles, Y., González-Mier, M. J., López-Hernández, B. E., \& Sánchez-Ojeda, E. . (2017). Derivaciones fecales y urinarias en un centro de atención especializado, México 2016. Enfermería universitaria, 14(4), 235-242. 
Research, Society and Development, v. 10, n. 7, e25410716442, 2021

(CC BY 4.0) | ISSN 2525-3409 | DOI: http://dx.doi.org/10.33448/rsd-v10i7.16442

Tibana, T. K., Fornazari, V. A. V., Gutierrez, J. W., Marchiori, E., Szejnfeld, D., \& Nunes, T. F. I. (2019). O que o radiologista deve saber sobre o papel da radiologia intervencionista na urologia. Radiol Bras ., 52 (5): 331-.336

Zapato-González, J. A., Camacho-Castro, J. B., García, S. M., Reyna-Bulnes, A. \& Robles-Scott, M. (2014). Nefrolitotomía percutánea en posición supina: nuestra experiencia. Revista de Urologia Mexicana. 74(6); 336-341. 